\title{
Is there a real need for a remotely actuated magnetic chest drain device?
}

\author{
Ludovic Fournel $^{1,2}$, Marco Alifano $^{1,2}$, Philippe Icard $^{1,3}$ \\ ${ }^{1}$ Department of Thoracic Surgery, Cochin Hospital, Paris Center University Hospitals, APHP, Paris, France; ${ }^{2}$ University of Paris, Paris, France; \\ ${ }^{3}$ University of Normandy, Caen, France \\ Correspondence to: Prof. Philippe Icard, MD, PhD. Thoracic Surgery Department of the Cochin Hospital, 27 rue du Fbg Saint Jacques 75014 , Paris, \\ France. Email: philippe.icard@aphp.fr. \\ Provenance: This is an invited article commissioned by the Section Editor Feichao Bao (Department of Thoracic Surgery, The First Affiliated \\ Hospital, Zhejiang University, Hangzhou, China). \\ Comment on: Taniguchi D, Matsumoto K, Kondo Y, et al. New Concept for a Thoracic Drainage System Using Magnetic Actuation. Surg Innov \\ 2019;26:705-11.
}

Submitted Nov 20, 2019. Accepted for publication Dec 01, 2019.

doi: $10.21037 /$ jtd.2019.12.60

View this article at: http://dx.doi.org/10.21037/jtd.2019.12.60

Thoracic drainage surgery has undergone significant changes over the past ten years. The "dogma" of systematic drainage by means of two tubes was initially called into question, except in special circumstances, such as in case of a significant risk of postoperative bleeding and major air sealing (1). An increasing number of teams have initiated enhanced rehabilitation programs after surgery (ERAS) to reduce postoperative morbidity as well as the hospitalization period. These protocols particularly include an optimization of perioperative nutrition, monitoring, physiotherapy, and, above all, patient mobilization as well as a removal of the chest tube as early as the situation allows it $(2,3)$. The generalization of ERAS programs has contributed to the evolution of the practices, the change of mentalities, and the global acceptance of the decreasing number of chest tubes insertions after lung surgery $(4,5)$. Interestingly, some authors go further in this direction and avoid insertion of chest tubes following limited or major pulmonary resection, and reported safe and reliable results $(6,7)$. Numerous studies have shown that it is also possible to reduce the duration of drainage as soon as the bleeding and air leak are not excessive. This has allowed a faster removal of drainage within the second day postoperative, or even in the hours following the intervention ("fast track"), with the objective of fast recovery and home discharge $(8,9)$.

As stated in the study by Taniguchi et al. (10), these changes in practice have been facilitated by the technical evolutions of drainage systems, whose development has been fostered by the inventiveness of the surgeons and by the support of the industry. The characteristics of the drains have evolved to become more flexible, and their calibre has been reduced; all this has resulted in a comfort increase (11). By draining along their whole length, some drains—similar to those used by Taniguchi et al.- - have seen their drainage capacity enhanced (12-14).

The setting of a "traditional" drain, perforated with a few orifices at its end, allows the drainage of fluid or air from a more limited part of the pleural cavity. From a radiological point of view, this may have the consequence of allowing only a sub-optimal pulmonary re-expansion, which has most often no symptomatic clinical expression. The perception of the impact of a persistent pleural gaseous or fluid effusion after pulmonary resection is rather "cultural"varying depending upon the surgeons and surgical teamsthan based on the likelihood of complications related to an incomplete pulmonary re-expansion (secondary infection of the pleural effusion, chest pain, aspergillus colonization...). Moreover, after anatomic lung resection, the production of pleural liquid is physiologically required and is useful to substitute the air in the remaining pleural cavity and could be useful to prevent minimal air leaks $(15,16)$.

In some residual effusions following surgery (as after extensive resection or treatment of pleuropulmonary infectious diseases) a single drainage system to avoid 
reinsertion of one or more chest tubes in the postoperative course, appears to be of great interest. Percutaneous chest drainage, "at patient's bedside", does not always allow the placement of the drainage device in an appropriate position. The procedure in itself scares the patients and it might sometimes be difficult for surgeons to achieve it. Similarly, ultra-sound- or CT-guided percutaneous drainage, when available, can also be painful and extend the length of hospital stay.

Due to its remote magnetic manipulation, the drain device dealt with in this article seems to provide an ingenious solution to the previously discussed issues, especially since its effectiveness has already been demonstrated in dry laboratory conditions and in a porcine model.

Nevertheless, some potential limitations need to be mentioned:

* Because of the formation of fibrinous or inflammatory adhesions, the pleural cavity tends to loculate relatively quickly, even in the absence of an infectious context. The capacity of the drain device to be efficiently moved under these conditions, and to "clear" the loculations, remains hypothetical and needs to be evaluated.

* The safety of the metal tip of such device should probably be tested over a longer period, in particular for the purpose of preventing accidents that could be ascribed to the placement of less flexible or rigid drain devices in the absence of vascular fistula or erosion risk (17).

* Similarly, the impact of the metal tip in terms of pain, whether at the time of insertion (percutaneous), during the drainage period or during removal should ideally be evaluated compared to the devices conventionally used.

Considering its widespread use supported by industrial production, the economic cost of acquiring and maintaining such a drainage system will have to be assessed and put into perspective with the actual clinical benefit compared to already existing devices.

Finally, it is necessary to remember that a large part of the residual effusions can be prevented by the proper positioning of the drain device by the surgeon at the end of the procedure, since he verifies that the drain remains in place during re-expansion of the lung before closing the intercostal space. For example, after an upper lobectomy, in order to drain the anterosuperior pleural space, a flexible chest tube must be placed so as to make a smooth curve in the upper thoracic area and then a few centimeters down the anterior wall. To prevent this very soft drain from falling into the cavity, it is better to keep it in good position by drawing it through a loose loop before attaching it to the parietal pleura (1). Thus, the prevention of the appearance of persistent fluid effusion relies, above all, on the careful verification of aerostasis and haemostasis at the end of the procedure.

To conclude, it is difficult at this stage to clearly identify the indications of this new type of flexible drain device modified at its end by a metal tip. At the end of a "standard" intervention, its location seems to be reduced, if the single drain has been placed correctly, if the hemostasis and the air sealing have been checked, it is rare-if not exceptionalto have to insert an additional chest tube, since a misplaced drainage device is usually inconsequential. The main indication of the drainage system proposed by Taniguchi et al. could be the percutaneous drainage of free or recently partitioned pleural effusions.

\section{Acknowledgments}

Céline QUINT a native English-speaking scientist of the Carré international-University of Caen Normandie which reviewed the language.

\section{Footnote}

Conflicts of Interest: The authors have no conflicts of interest to declare.

Ethical Statement: The authors are accountable for all aspects of the work in ensuring that questions related to the accuracy or integrity of any part of the work are appropriately investigated and resolved.

\section{References}

1. Icard P, Chautard J, Zhang X, et al. A single 24F Blake drain after wedge resection or lobectomy: a study on 100 consecutive cases. Eur J Cardiothorac Surg 2006;30:649-51.

2. Van Haren RM, Atay SM. Enhancing the study of enhanced recovery after thoracic surgery: methodology and population-based approaches for the future. J Thorac Dis 2019;11:S612-8.

3. Medbery RL, Fernandez FG, Khullar OV. ERAS and patient reported outcomes in thoracic surgery: a review of 
current data. J Thorac Dis 2019;11:S976-86.

4. Okur E, Baysungur V, Tezel C, et al. Comparison of the single or double chest tube applications after pulmonary lobectomies. Eur J Cardiothorac Surg 2009;35:32-5; discussion 35-6.

5. Brunelli A, Beretta E, Cassivi SD, et al. Consensus definitions to promote an evidence-based approach to management of the pleural space. A collaborative proposal by ESTS, AATS, STS, and GTSC. Eur J Cardiothorac Surg 2011;40:291-7.

6. Ueda K, Haruki T, Murakami J, et al. No Drain After Thoracoscopic Major Lung Resection for Cancer Helps Preserve the Physical Function. Ann Thorac Surg 2019;108:399-404.

7. Lu TY, Chen JX, Chen PR, et al. Evaluation of the necessity for chest drain placement following thoracoscopic wedge resection. Surg Today 2017;47:606-10.

8. Deng B, Qian K, Zhou JH, et al. Optimization of Chest Tube Management to Expedite Rehabilitation of Lung Cancer Patients After Video-Assisted Thoracic Surgery: A Meta-Analysis and Systematic Review. World J Surg 2017;41:2039-45.

9. Eustache J, Ferri LE, Feldman LS, et al. Enhanced recovery after pulmonary surgery. $\mathrm{J}$ Thorac Dis 2018;10:S3755-60.

10. Taniguchi D, Matsumoto K, Kondo Y, et al. New

Cite this article as: Fournel L, Alifano M, Icard P. Is there a real need for a remotely actuated magnetic chest drain device? J Thorac Dis 2019;11(12):5677-5679. doi: 10.21037/jtd.2019.12.60
Concept for a Thoracic Drainage System Using Magnetic Actuation. Surg Innov 2019;26:705-11.

11. Nakamura H, Taniguchi Y, Miwa K, et al. The 19Fr Blake drain versus the $28 \mathrm{Fr}$ conventional drain after a lobectomy for lung cancer. Thorac Cardiovasc Surg 2009;57:107-9.

12. Sakakura N, Fukui T, Mori S, et al. Fluid drainage and air evacuation characteristics of Blake and conventional drains used after pulmonary resection. Ann Thorac Surg 2009;87:1539-45.

13. Niwa Y, Koike M, Oya H, et al. Method of bilateral pleural drainage by single Blake drain after esophagectomy. World J Surg 2015;39:727-31.

14. Saxena P, Kejriwal N, Newman MA. Use of Blake drains following lung resection. Eur J Cardiothorac Surg 2006;30:952; author reply 952-5.

15. Refai M, Brunelli A, Salati M, et al. The impact of chest tube removal on pain and pulmonary function after pulmonary resection. Eur J Cardiothorac Surg 2012;41:820-2; discussion 823.

16. Sziklavari Z, Neu R, Hofmann HS, et al. Persistent pleural effusion following thoracic surgery. Chirurg 2015;86:432-6.

17. Coulter TD, Maurer JR, Miller MT, et al. Chest wall arteriovenous fistula: an unusual complication after chest tube placement. Ann Thorac Surg 1999;67:849-50. 